\title{
Chitosan-based coatings in the prevention of intravascular
}

\section{catheter-associated infections}

\section{Gracia Mendoza, ${ }^{1}$ Anna Regiel-Futyra, ${ }^{2}$ Alejandra Tamayo, ${ }^{1}$ Marta Monzon, ${ }^{3}$ Silvia Irusta, ${ }^{1}$ Miguel A. de Gregorio, ${ }^{4}$ Agnieszka Kyziol, ${ }^{2}$ Manuel Arruebo ${ }^{1}$}

${ }^{1}$ Department of Chemical Engineering, Aragon Institute of Nanoscience (INA), University of Zaragoza, Campus Río Ebro-Edificio I+D, C/ Mariano Esquillor S/N, 50018 Zaragoza, Spain; Networking Research Center on Bioengineering, Biomaterials and Nanomedicine, CIBER-BBN, Madrid 28029, Spain; Aragon Health Research Institute (IIS Aragón), 50009 Zaragoza, Spain

${ }^{2}$ Faculty of Chemistry, Jagiellonian University, Ingardena 3, 30-060 Kraków, Poland.

${ }^{3}$ Research Centre for Encephalopathies and Transmissible Emerging Diseases, Universidad de Zaragoza, 50013 Zaragoza, Spain

${ }^{4}$ Networking Research Center on Bioengineering, Biomaterials and Nanomedicine, CIBERBBN, Madrid 28029, Spain, and Grupo de Investigación en Técnicas de Mínima Invasión (GITMI) del Gobierno de Aragón, Universidad de Zaragoza, Zaragoza, Spain

Corresponding author: Gracia Mendoza, University of Zaragoza, Campus Río EbroEdificio I+D, C/ Mariano Esquillor S/N, 50018 Zaragoza, Spain Email: gmmenc@unizar.es 


\section{Abstract}

Central venous access devices play an important role in patients with prolonged intravenous administration requirements. However, these medical devices have shown to be susceptible to develop microbial infections with the associated risk of affecting the whole organism. In this sense, coating these devices with bactericidal compounds has emerged as a potential tool to prevent bacterial colonization. Our study describes the modification of 3D-printed reservoirs and silicone catheters, mimicking central venous access devices, through different approaches including their coating with the well-known biocompatible and bactericidal polymer chitosan, with the anionic polysaccharide alginate and also plasma treated surfaces were also included in the study to promote polymer adhesion. The evaluation of the antimicrobial action of those surface modifications compared to that exerted by a model antibiotic (ciprofloxacin) adsorbed on the surface of the devices was carried out. Surface characterization was developed by different methodologies and the bactericidal effects of the different coatings were assayed in an in vitro model of Staphylococcus aureus infection. Our results showed the great importance of the surface device topography for the successful attachment of the coating and for the subsequent development of bactericidal effects. Furthermore, the single presence of chitosan was enough to fully inhibit bacterial growth exerting a comparable efficiency as that showed by the model antibiotic though avoiding the potential emergence of antibiotic mediated resistances. Importantly, chitosan coating showed low cytotoxicity against human keratinocytes, human lung adenocarcinoma epithelial cells and murine colon carcinoma cells. Chitosan-based coatings are proposed as an effective and promising solution in the prevention of microbial infections associated to medical devices.

Keywords: Central venous access devices, intravascular, bacterial infections, chitosan, antimicrobial 


\section{Introduction}

Central venous access devices (CVADs), also known as implantable ports, are of common use in the clinic and have become in a mainstay for patients who require prolonged intravenous drugs or nutrients administration. ${ }^{1,2}$ These devices consist of a catheter and a surgically implantable reservoir which are inserted in central veins providing long-term implantation unlike peripheral catheters. ${ }^{3}$ However, CVADs are prone to develop contamination resulting in chronic and nosocomial infections which can potentially be harmful due to their access to the whole organism through principal veins and may lead to major complications and to the associated high health-care burden. ${ }^{4}$ The site of insertion may initiate of microbial infection favoring the colonization of the catheter and the implanted reservoir. ${ }^{5}$ Furthermore, CVADs may be obstructed by thrombotic processes produced mainly inside or around the catheters, which can even impair their functionality. ${ }^{3,6}$ To solve these issues, coating the catheters with different materials such as Hydromer (polyvinylpyrrolidone) has been demonstrated as effective in the reduction of thrombosis and bacterial colonization. ${ }^{7,8}$

Staphylococcus aureus has been reported to be present in different types of infected and noninfected wounds. ${ }^{9}$ Furthermore, it has been shown as highly prevalent and problematic in different wound infections, e.g. surgical wounds, recognizing its high incidence not only in wounds but also in nosocomial infections. ${ }^{9}$ Regarding medical devices, $S$. aureus may impair the functionality of implanted stents ${ }^{10}$ and plays an important role in the development of nosocomial infections of implants, catheters, devices, shunts, valves or prostheses. ${ }^{1}$ Its pathogenicity and its capacity to form biofilm represents an additional hurdle in the treatment of infections associated to medical devices. ${ }^{11}$ In this sense, it has been experimentally assayed both in vitro and in vivo as prevalent microorganism to mimic biofilm or nosocomial 
infections $^{12,13}$ or even when treating $S$. aureus infections with antibiotics as a model ${ }^{14,15}$ or with other compounds. ${ }^{16}$

To avoid these infections, antibiotic prophylaxis has been shown as ineffective. ${ }^{17}$ This fact together with the possible generation of antibiotic resistance ${ }^{2,5}$ points towards the need of developing new approaches such as the use of bactericidal coatings on those devices to avoid microorganism colonization.

The coating of catheters with different compounds such as cefazolin ${ }^{18}$, ifampicin $^{19}$, silver $^{10,20}$ and chlorhexidine-silver sulfadiazine ${ }^{21}$ has been reported with similar efficiency against bacterial colonization though the potential development of antibiotic resistance impairs their antimicrobial capacity being more susceptible to develop infections than any antiseptic coating. ${ }^{21}$ In spite of the large antimicrobial action of silver, it has also been demonstrated its potential allergenic induction, cytotoxicity, induction of inflammation, apoptosis and DNA damage probably due to the release of silver ions ${ }^{22,23}$ together with the development of bacterial resistances. ${ }^{24,25}$ However, several commercial silver-coated catheters are being used in the clinical practice based on bio-inert silver-based zeolite (Vygon $\left.{ }^{\circledR}\right)$ or on the galvanic effect mediated by the combination of silver, gold and palladium (Bactiguard $\AA$ ), though recent studies have shown no differences in the development of microorganisms growth in silver-coated and non-coated catheters obtained from catheterized patients. ${ }^{26}$ Furthermore, the coating of polydimethylsiloxane substrates with monomeric trimethylsilanes, including or not oxygen during the deposition procedure, has also been shown as an effective system to reduce the development of $S$. aureus associated infections both in vitro and in vivo. ${ }^{11}$ In this sense, the coating of the reservoir surface with biocompatible polymeric layers or films has emerged as an attractive approach. ${ }^{27}$ Another interesting surface modification, combining both chemical and physical modifications, is oxygen plasma treatment. In this process, a biomaterial surface in contact with plasma is bombarded with several species such as 
electrons, photons, ions or free radicals, leading in consequence to surface cleaning, ablation and changes in the chemical composition of the substrate without altering its bulk properties. ${ }^{28}$ Moreover, the incorporation of oxygen containing functional groups, needed for drug or other polymer bonding, can be easily achieved after bombardment ${ }^{29}$ and this treatment can also reduce bacterial adhesion. ${ }^{28}$ This method has been successfully applied for the modification of polymeric surfaces..$^{28,29}$

In order to create an ideal biocompatible, anti-adhesive, anti-fouling and anti-infective surface on an implant, it is desirable to reduce bacterial adhesion by modifying the medical devices with bacteriostatic or antibacterial polymers such as chitosan (CS) or alginate (AG). ${ }^{29-32} \mathrm{CS}$ is a polycationic aminopolysaccharide obtained by partial N-deacetylation of chitin, a natural biopolymer derived from crustacean shells, shrimps or other microorganisms such as fungi. ${ }^{33}$ In contrast to chitin, its derivative is soluble in most dilute acidic solutions at pHs below 6.5 due to the quaternisation of the amine groups with a pKa value of $\sim 6.3 .^{34}$ The protonation of these functional groups at $\mathrm{pH}$ below the $\mathrm{pKa}$ makes $\mathrm{CS}$ a water-soluble polyelectrolyte, which solubility strongly depends on the solvent $\mathrm{pH}$. Another important parameter influencing the functional and physiological activity of $\mathrm{CS}$ is the average molecular weight $(\mathrm{Mw}){ }^{35}$ Depending on the polymer origin and the choice of the deacetylation process conditions, different deacetylation degrees and molecular weights can be obtained altering the resulting physicochemical and biological properties. In fact, moderate molecular weights and high deacetylation degrees have been shown higher bactericidal and anti-biofilm effects compared to low degrees and low $\mathrm{Mw}$ and oligomers ${ }^{36,37}$ though the correlation between the CS Mw and its antimicrobial action is not clearly defined. Over the last decades, CS has attracted a huge interest, mainly due to its biocompatibility, biodegradability and antimicrobial activity. ${ }^{38,39}$ Its low toxicity profile combined with inertness have been proven by numerous in vitro and in vivo studies, reporting the lack of 
inflammatory or allergic reactions resulting from implantation, injection or topical application on the human body. ${ }^{40} \mathrm{CS}$ exhibits a wide range of antibacterial and antifungal activity $^{41}$ thus several antimicrobial CS-based products are commercially available ${ }^{39,42,43}$ though the exact antimicrobial mechanism has not been fully elucidated yet. The most convincing hypothesis relates to the polycationic nature of CS and the resulting interactions between protonated amino groups and negatively charged components of microorganism cell walls, though the binding of CS to teichoic acids and the potential extraction of membrane lipids have also been proposed as potential mechanisms responsible for its pathogenicity. ${ }^{44}$ Unlike CS, sodium AG is a negatively charged polysaccharide obtained from brown sea weed, readily soluble in water. Similarly to CS, sodium AG has gained a lot of attention due to its unique properties such as biocompatibility, biodegradability, non-toxicity and low cost. ${ }^{45} \mathrm{AG}$ undergoes a mild gelation under the addition of divalent ions such as $\mathrm{Ca}^{2+}$, leading to hydrogel formation which can also be prepared by other cross-linking methods. ${ }^{46}$ Due to its features, it has been successfully used in tissue regeneration ${ }^{47}$ and drug delivery applications. ${ }^{48}$ Commercially available sodium alginates can vary in the molecular weight, which determines their physical properties. It is postulated that as the $\mathrm{Mw}$ increases, an improvement in the mechanical properties can be obtained, though the higher Mw, the higher viscosity and consequently its processability gets impaired.

The present work proposes the use of dip coating techniques to coat 3D-printed reservoirs and silicone catheters with the bactericidal and biocompatible polymers CS and sodium AG, and with the model antibiotic, ciprofloxacin. In some cases an oxygen plasma treatment was also applied in order to increase the polymer or ciprofloxacin deposition and to reduce bacterial attachment. The antibacterial effectiveness of the different coatings assayed was investigated against $S$. aureus to elucidate the most suitable antimicrobial surface in order to avoid bacterial colonization of CVADs. 


\section{Materials and methods}

\section{Chitosan, sodium alginate and ciprofloxacin hydrochloride solutions preparation}

Chitosan with medium average molecular weight (deacetylation degree (\%) $89 \pm 2$; viscosity 200-800 cP, 1 wt. $\%$ in $1 \%$ acetic acid $\left.25^{\circ} \mathrm{C}\right)^{49}$, sodium alginate and ciprofloxacin hydrochloride were purchased from Sigma Aldrich (Germany). Acetic acid (99.8\%), used as a solvent, and sodium hydroxide were also supplied by Sigma Aldrich (Germany). All solutions were prepared in deionized water (MiliQ).

CS stock solution $(1 \%(\mathrm{w} / \mathrm{v}))$ was prepared by dissolving polymer flakes in an aqueous solution of acetic acid $(0.1 \mathrm{M})$. Heating $\left(65^{\circ} \mathrm{C}\right)$ and mixing was maintained until a clear solution was obtained (around 12h). The pH of CS solution was $\sim 4.5$. Sodium AG $2 \%(w / v)$ solution was prepared in MiliQ water at room temperature. Ciprofloxacin hydrochloride was dissolved in MiliQ water and in the previously prepared $1 \%(\mathrm{w} / \mathrm{v})$ CS solution to obtain the concentration of $100 \mu \mathrm{g} / \mathrm{mL}$. The stability of the antibiotic in acidified polymer solution was monitored after several days by UV-Vis measurements. Sodium hydroxide $1 \%(\mathrm{w} / \mathrm{v})$ aqueous solution was used for the neutralization of CS-coated scaffolds.

\section{Reservoirs and catheters}

The 3D design and printing of the reservoirs were developed with a 3D printer Objet Eden $350 \mathrm{~V}$ using a methacrylate derivative (proprietary composition) (Stratasys, US). The original CVAD and the autoCAD image used as model for the 3-D printer are shown in Figure 1. The silicone tubing, with the external diameter $4 \mathrm{~mm}$, was purchased from MCZ (Poland). Catheters and 3D-printed scaffolds obtained are also presented in Figure 1. 

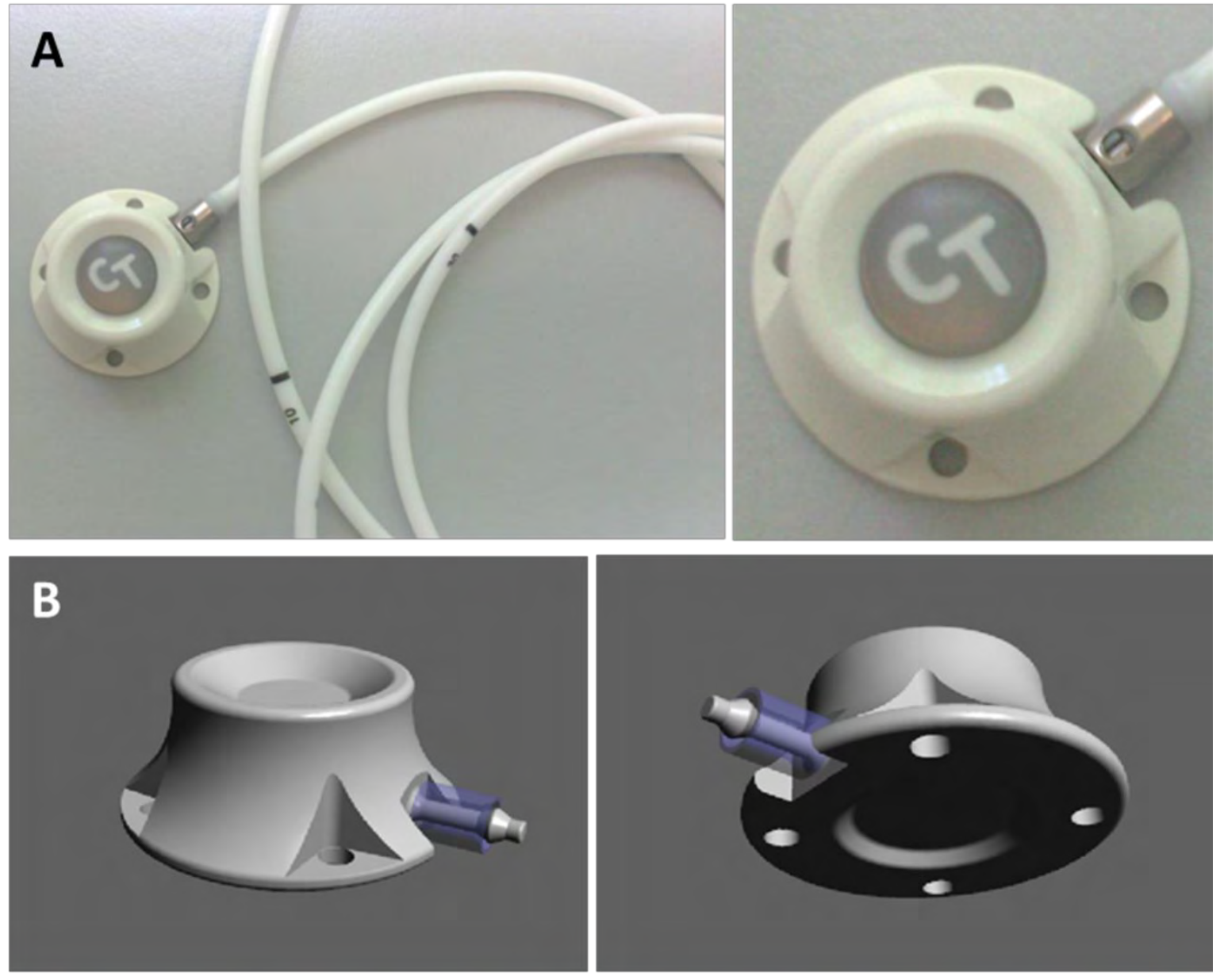

C 
Figure 1. CVAD design. (A) Original CVAD for clinical use (Port-A-Cath ${ }^{\circledR}$, Smiths Group plc.). (B) CVAD 3D-design for our prototype. (C) CVAD prototype obtained from a 3D printer from top (left) and bottom (middle) views, and silicon catheter.

\section{Dip coating and characterization}

The dip coating technique consisted on the immersion of the catheters and reservoirs in the liquid coating medium (solubilized chitosan) followed by the evaporation of the solvent and the consequent deposition of the film on the surface of the catheters and reservoirs used as models. ${ }^{50}$

The coating of the scaffolds with the polymers was performed with and without oxygenplasma treatment before dip-coating. A low pressure, low temperature plasma treatment was applied to remove the impurities and to generate oxygen-containing functional groups on the scaffolds surface. An enhancement in the polymer adhesion thanks to the presence of oxygen groups on the treated scaffold was expected. ${ }^{28,29}$ An oxygen flow (99.5\%, Air Products) at a pressure of $<0.3$ mbar was introduced to the generator chamber. $100 \mathrm{~W}$ power and $40 \mathrm{~Hz}$ frequency were applied during the $30 \mathrm{~s}$ of the plasma treatment. In the experiment a Zepto plasma generator (Diener Electronic Plasma Technology, US) and a Dip Coater TLO.01 (MTI Corporation, US) were used. The speed of the sample withdrawal from the solution was 
$1 \mathrm{~cm} / \mathrm{min}$. Silicone tubes and reservoirs were thoroughly cleaned with water and ethanol (Sigma Aldrich, 99.5\% purity, Germany) and dried before coating. All of the scaffolds were weighed before and after coating in order to evaluate the weight gain. The number of group samples and coating protocols are presented in Scheme 1. Each group was composed of three samples to reach statistical significance.

In each case, after dip coating with the corresponding polymer or antibiotic solution, samples were gently dried with a heat gun. In order to exclude any potential influence of the CS solvent (diluted acetic acid) on the antibacterial activity, two ways of neutralization were also conducted. 3D scaffolds and silicone tubes were submerged in $1 \%(\mathrm{w} / \mathrm{v})$ sodium hydroxide solution and in deionized water (Scheme 1.11) after coating or the CS solution $\mathrm{pH}$ was adjusted to 5.73 (Scheme 1.12) before coating.

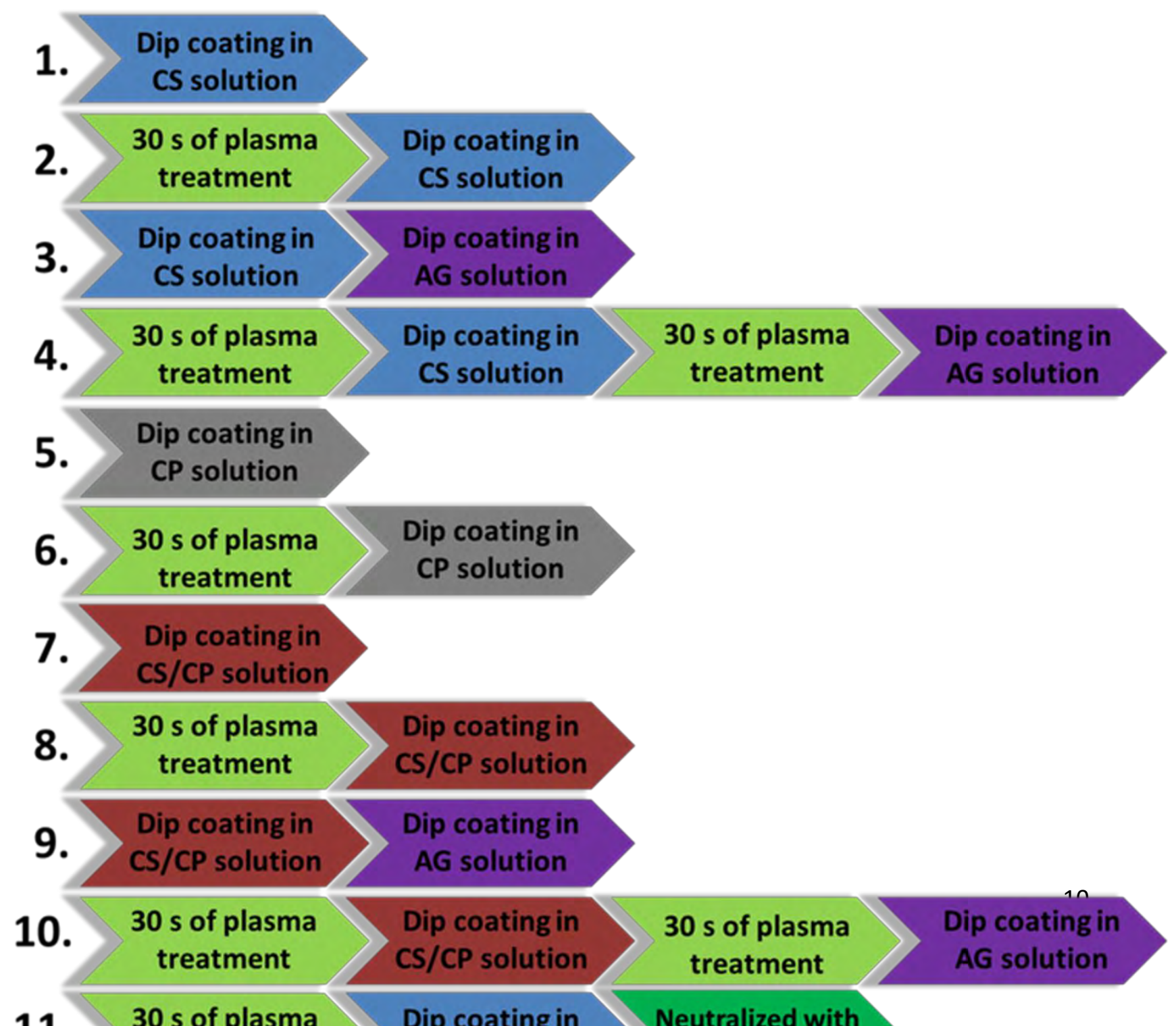


Scheme 1. Types of surface modifications on the CVADs prototypes and on the silicon tubes $(\mathrm{CS}=$ Chitosan; $\mathrm{AG}=$ Alginate $\mathrm{CP}=$ Ciprofloxacin $)$.

The surface morphology of the reservoirs and tubes before and after coating was observed using scanning electron microscopy (SEM, Quanta FEG-250, FEI, US). The characterization of the surface roughness and waviness was carried out in a Kla-Tencor P6 Stylus Profiler (US). The chemical characterization of the surface before and after CS coating was performed by X-ray photoelectron spectroscopy (XPS) by using an Axis Ultra DLD (Kratos Analytical, UK). A monochromatic Al $\mathrm{K} \alpha \mathrm{X}$-ray radiation $(\mathrm{h} v=1486.6 \mathrm{eV})$ was used as excitation source at $15 \mathrm{kV}$ and $10 \mathrm{~mA}$. The peaks analysis was developed by the CasaXPS software (Casa Software Ltd, UK). Fourier transform infrared spectroscopy (FTIR) was carried out to study surface infrared absorption of CS coated and uncoated reservoirs in a Bruker Vertex 70 (Bruker Corporation, US) equipped with a DTGS detector and ATR Golden Gate Diamond. The spectra were obtained with $4 \mathrm{~cm}^{-1}$ resolution in a range $4000-400$ $\mathrm{cm}^{-1}$. 
In addition, the stability of ciprofloxacin hydrochloride in water and in CS acidified solution was evaluated by UV-Vis measurements (UV-Vis spectrophotometer Perkin Elmer, Lambda 35 , US) in order to check the chemical integrity of the molecule.

\section{In vitro infection model}

Staphylococcus aureus (BAControl, Ielab, Spain) was chosen as model bacteria due to its prevalence in device-associated infections as explained above. Lyophilized bacteria were reconstituted in tryptone soy broth (TSB) medium (Cultimed, Panreac, Spain) and further cultured overnight in the same medium at $37^{\circ} \mathrm{C}$ with agitation $(150 \mathrm{rpm})$.

Prior to the biological assays, reservoirs and catheters were sterilized by gas plasma (Sterilization facility, Hospital Clínico Universitario Lozano Blesa, Zaragoza, Spain) to assure the adequate course of the infection model avoiding contaminations as well as catheters were cap-ended with stoppers to ensure that bacteria only contacted with the coated surfaces.

The efficiency of the different coatings developed against infections (Scheme 1) was tested against an overnight $S$. aureus culture diluted to obtain $10^{2} \mathrm{CFU} / \mathrm{mL}$ in fresh TSB medium. We selected this bacterial count rate because according to the clinical practice guidelines for the diagnosis and management of intravascular catheter-related infections, a bacterial count rate above $10^{2} \mathrm{CFU} / \mathrm{mL}$ (determined from a catheter by quantitative (sonication) broth culture) reflects catheter colonization ${ }^{51}$. The reservoirs and catheters were independently placed in the bottom of sterilized bottles and the diluted bacteria culture added to a volume of $40 \mathrm{~mL}$ in order to completely cover them. Samples were incubated overnight at $37{ }^{\circ} \mathrm{C}$ with agitation $(150 \mathrm{rpm})$. Then, samples aliquots collected from the bacteria culture were diluted in sterilized PBS and grown overnight on tryptone soy agar plates at $37^{\circ} \mathrm{C}$. After incubation, $S$. aureus colonies were counted and the data expressed as CFU/mL standardized to the coating weight $(\mathrm{mg})$. Control samples were also run to monitor the development of the 
experiments. These control samples were a positive control sample in which a non-coated reservoir/catheter was incubated with $S$. aureus suspension to check normal bacteria colonization, a negative control consisting of a coated reservoir/catheter incubated in TSB without bacterial inoculum to check the adequate sterilization process, and different control samples without reservoir/catheter $(\mathrm{TSB}+S$. aureus; TSB + Ciprofloxacin; TSB + S. aureus + Ciprofloxacin) in order to test the methodology. Each group consisted of three independent samples and tests were performed in triplicate obtaining nine data per group to verify the reproducibility of the assays.

\section{In vitro cytotoxicity test}

Thiazolyl Blue Tetrazolium Bromide (MTT, 98\%), Resazurin Sodium Salt (Alamar Blue), DMSO and methanol were supplied by Sigma Aldrich (Germany). Phosphate-Buffered Saline (PBS) without $\mathrm{Ca}^{2+}$ and $\mathrm{Mg}^{2+}$, fetal bovine serum (FBS), trypsin and antibiotics (Penicillin/Streptomycin/Amphotericin B Mix) were purchased from Pan Biotech (Germany). Dulbecco's Modified Eagle Medium (DMEM) with $4.5 \mathrm{~g} / \mathrm{L}$ glucose, with L-Glutamine, sodium pyruvate and Phenol Red, supplied by Corning (US), was used in cell culturing. To determine the cytotoxic activity of the CS coatings, three different cell lines were used in this study: an immortal human keratinocyte (HaCaT), human lung adenocarcinoma epithelial cell line (A549) and murine colon cancer cell line (CT26). Cells were maintained in high-glucose DMEM with $1 \%$ of antibiotics and $10 \%$ of FBS. Cells were cultured at $37{ }^{\circ} \mathrm{C}$ in $5 \% \mathrm{CO}_{2}$ saturated air. Culture media were replaced every 2 days. Cells were passaged at least once a week.

In vitro biocompatibility of the CS coatings was determined using material extracts. ${ }^{52} \mathrm{CS}$ layers were prepared by pouring the CS solution into 12-well plates and drying in an electric oven at $60{ }^{\circ} \mathrm{C}$ for $\sim 3 \mathrm{~h}$ followed by neutralization with 1 wt. $\% \mathrm{NaOH}$ and deionized water. Before the experiment, chitosan layers were sterilized under UV light for 30 minutes. The 
extracts were prepared as follows: each well with the chitosan layer was treated with $1.5 \mathrm{~mL}$ of cell culture medium without FBS and incubated for $24 \mathrm{~h}\left(37^{\circ} \mathrm{C}, 5 \% \mathrm{CO}_{2}\right.$ atmosphere). Cells were seeded in 24 -well flat bottom plates and also incubated for $24 \mathrm{~h}\left(37^{\circ} \mathrm{C}, 5 \% \mathrm{CO}_{2}\right.$ atmosphere) to obtain a final density of $7.5 \times 10^{4}$ cells/well. After $24 \mathrm{~h}$ the medium was replaced with the material extract at the concentration $100 \%$ or $50 \%$ when diluted with DMEM medium enriched with 10\% FBS. Cells treated with DMEM without (S(-)) and with $10 \%$ FBS $(\mathrm{S}(+))$ were used as a positive control. After $24 \mathrm{~h}$ incubation cell viability was determined by the MTT and Alamar Blue assays ${ }^{53}$. Briefly, each well was rinsed with PBS and treated with $250 \mu \mathrm{L}$ of the MTT solution $(0.5 \mathrm{mg} / \mathrm{mL})$ or Alamar Blue solution $\left(25 \times 10^{-6}\right.$ M) and incubated at $37^{\circ} \mathrm{C}$ for $3 \mathrm{~h}$. In MTT assay, insoluble purple formazan crystals were dissolved in DMSO: $\mathrm{CH}_{3} \mathrm{OH}(1: 1)$. The absorbance at $570 \mathrm{~nm}$ was measured in a microplate reader (Infinite $200 \mathrm{M}$ PRO NanoQuant, Tecan) with a reference wavelenght at $670 \mathrm{~nm}$. Results are presented as surviving cells fraction, $\left[\left(\mathrm{A}_{570 \mathrm{~nm}}\right.\right.$ of treated cells $\left.-\mathrm{A}_{670}\right) /\left(\mathrm{A}_{570 \mathrm{~nm}}\right.$ of untreated cells - $\mathrm{A}_{670} \times 100 \%$, and expressed as mean values \pm standard deviation. In the Alamar Blue assay, the fluorescence measurements were recorded with the $\lambda_{\text {ext }}$ at $560 \mathrm{~nm}$ and $\lambda_{\mathrm{em}}$ at $605 \mathrm{~nm}$. Results are presented as surviving cells fraction and expressed as mean values \pm standard deviation. The results are the average values of three independent experiments.

\section{Statistical analyses}

Results are reported as mean \pm SEM. Statistical study of data was performed using the StataSE 12 statistical software (StataCorp LP, US). The normal distribution of the variables was analyzed by the Shapiro Wilk test followed by ANOVA test, t-Student test or U-Man Whitney test. $\mathrm{P} \leq 0.05$ was considered to be statistically significant.

\section{Results and discussion}

\section{Surface characterization}


SEM micrographs of the surface of the uncoated reservoirs exhibit a differentiated topography (Figure 2). The coating of the scaffolds with polymers, both CS and CS/AG, significantly decreased the irregularity of the substrate and a smooth nanostructure resulted from the polymer presence was observed after coating. No significant differences between samples with and without plasma treatment were observed. SEM pictures of silicone tubes did not reveal any difference in the surface topography before and after coating with polymers irrespectively of the plasma treatment used (Figure 3).

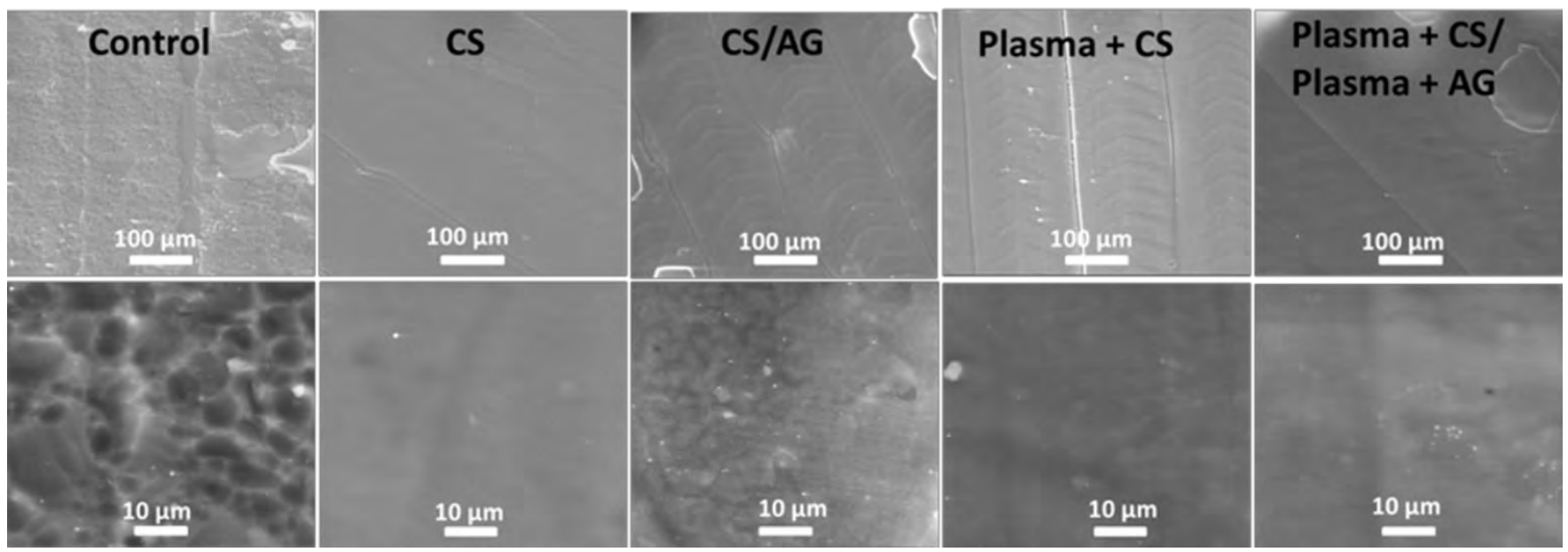

Figure 2. SEM micrographs of the 3D-printed reservoirs before (Control) and after coating with chitosan (CS - Group 1), chitosan and alginate (CS/AG - Group 3), chitosan after 
plasma treatment (Plasma $+\mathrm{CS}-$ Group 2), chitosan and alginate after plasma treatment steps (Plasma + CS/ Plasma + AG - Group 4).
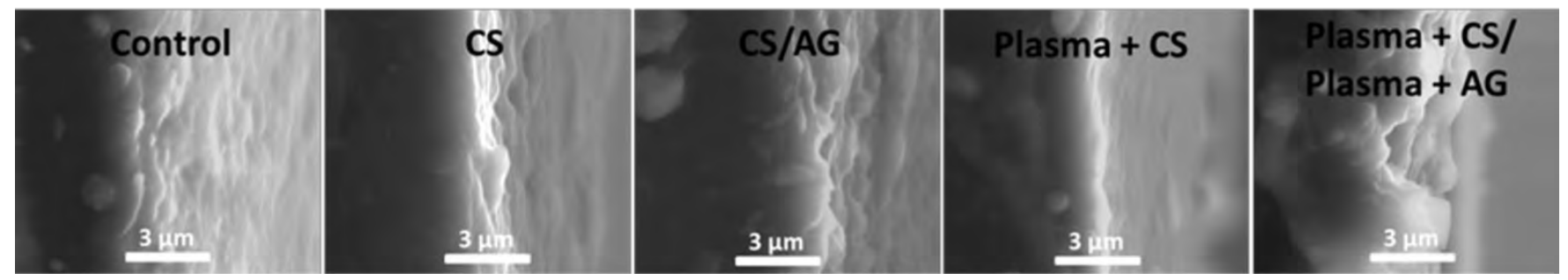

Figure 3. SEM micrographs of silicone tubes before (Control) and after coating with chitosan (CS- Group 1), chitosan and alginate (CS/AG - Group 3), chitosan after plasma treatment (Plasma + CS - Group 2), chitosan and alginate after plasma treatment steps (Plasma + CS/ Plasma + AG - Group 4).

In order to further assess the nanostructure of the reservoirs and catheters before and after coating, their roughness profiles were also analyzed (Table 1). The surface length studied was $500 \mu \mathrm{m}$ in the case of the reservoirs and $200 \mu \mathrm{m}$ for the catheters. These results pointed to a significant higher surface roughness of the uncoated reservoirs (control samples) than that obtained for the uncoated silicone tubes (control samples) being the reservoirs roughness a $70 \%$ higher than that displayed for the catheters which might determine the coating adherence and efficiency and thus the subsequent bactericidal effects. After coating, reservoirs exerted a statistically significant reduction (58-73\%) in surface roughness not finding statistically significant changes among the different coatings. However, catheters did not significantly vary their roughness values and no significant differences between coated and non-coated samples were found. 
Table 1. Roughness profiles of the reservoirs and catheters before (Control) and after plasma treatment and CS and AG coating. The results are expressed as mean $\pm \mathrm{SD}(\mathrm{nm})$ of at least three independent experiments

\begin{tabular}{|c|c|c|}
\hline & TREATMENT & ROUGHNESS (nm) \\
\hline \multirow{5}{*}{ 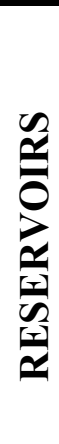 } & Control & $25.0 \pm 3.0$ \\
\hline & CS & $10.5 \pm 2.5$ \\
\hline & CS/AG & $7.0 \pm 1.7$ \\
\hline & Plasma CS & $9.5 \pm 2.2$ \\
\hline & Plasma CS/AG & $8.8 \pm 2.0$ \\
\hline \multirow{2}{*}{ 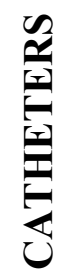 } & Control & $7.5 \pm 1.6$ \\
\hline & CS & $7.7 \pm 1.7$ \\
\hline
\end{tabular}

The determination of surface elemental concentrations for non-coated and CS coated reservoirs was performed by using XPS analysis (Table S1). The atomic composition of the reservoir surface showed that the CS coating increased the N/C ratio from 0.008 to 0.058 due to the $\mathrm{N} 1 \mathrm{~s}$ atomic percentage increase from 0.7 at. $\%$ to 4.54 at. $\%$ after CS coating. This nitrogen increase is caused by the incorporation of primary amino groups from the chitosan backbone. The high resolution deconvolution of the $\mathrm{N}$ 1s peaks (data not shown) revealed a single peak at $400 \mathrm{eV}$ for the control reservoirs indicative of the presence of R-CN groups ${ }^{54}$. However, the CS coated reservoirs displayed two other peaks reducing the atomic percentage of $\mathrm{R}-\mathrm{CN}$ in coated reservoirs until $18 \%$. The new peaks found were $\mathrm{R}^{-\mathrm{NH}_{2}}(67 \%)$ and $\mathrm{R}-$ $\mathrm{NH}_{3}{ }^{+}(15 \%)$, which clearly demonstrate the CS coating on the reservoirs. 
To further characterize the reservoir surface regarding the interactions between the functional groups of the reservoir material and the CS, FTIR spectra of pure CS and reservoirs before and after CS coating were studied (Table S2). CS spectrum shows its characteristic bands at around $1640 \mathrm{~cm}^{-1}$ and $1590 \mathrm{~cm}^{-1}$ which is consistent with amide and amine groups, respectively ${ }^{49}$. Uncoated reservoirs spectra show a band at $1725 \mathrm{~cm}^{-1}$ related to the stretching vibration of $\mathrm{C}=\mathrm{O}$ groups ${ }^{55}$, and bands at 1638 and $1450 \mathrm{~cm}^{-1}$ assigned to acrylate double bonds and distortion vibrations of $\mathrm{CH}_{2}$ respectively ${ }^{56}$. After $\mathrm{CS}$ coating, the presence of a new band at $1568 \mathrm{~cm}^{-1}$, due to the asymmetric stretching of carboxylic groups, indicated the interaction of the CS amine groups with the carboxylic groups of the acrylonitrile present in the reservoirs ${ }^{57}$. These data evidenced the successful deposition of CS coating onto the reservoir through the reaction of the carboxylic groups of the reservoir acrylate and the CS amine groups. The XPS and FTIR spectra of the CS coated silicone tubes did not indicate the presence of the CS (data not shown). These results, together with the lack of surface roughness modification indicate that $\mathrm{CS}$, under the tested conditions, did not adhere to the silicone tubes used as models.

Finally, to test the stability of the model antibiotic assayed in the CS acidic solution, changes in the absorption spectra were also recorded. UV-Vis spectra of ciprofloxacin hydrochloride after dissolution in acidified chitosan did not show changes in comparison to distilled water at both time points ( 0 and 4 days) assayed (data not shown) indicative of the stability of the compound in acidic solutions.

\section{In vitro bacterial growth}

The measurement of the inhibition of $S$. aureus growth mediated by the coated scaffolds and catheters was evaluated by counting the number of $\mathrm{CFU} / \mathrm{mL}$ obtained from the bacteria culture after overnight incubation in a $10^{2} \mathrm{CFU} / \mathrm{mL}$ bacterial solution and further growth in agar plates. The results obtained for some of the scaffolds and catheters assayed are depicted 
in Figure 4 and in Figure 5, respectively. It was found that for the reservoirs the presence of CS in the coating was enough to exert a significant and potent bactericidal effect and no $S$. aureus growth was displayed (Figure 4) which was corroborated after plating and counting. In fact, CS coatings on any of substrate modifications used were as efficient as ciprofloxacin adsorbed on the reservoirs which is a very relevant and notable data in a potential clinical application in order to avoid antibiotic-associated resistances. With this efficient bactericidal response exerted by the CS by itself no differences were observed when the substrate was previously treated with plasma, when it was re-coated with AG or even when it was embedded with CS and ciprofloxacin. In fact, the efficiency and homogeneity of the coating was corroborated due to the lack of bacterial growth along the device because any uncoated area would constitute a surface prone to bacterial colonization. 
Figure 4. Scaffolds after treatment with $S$. aureus $\left(10^{2} \mathrm{CFU} / \mathrm{ml}\right)$. Positive and negative control samples are displayed in both pictures showing the bacteria growth in positive control as the CVAD was not coated and treated with the microorganism while the negative control was not treated with $S$. aureus showing that the sterilization process was successful for this type of materials. The experimental groups assayed did not show S. aureus growth due to the coating (chitosan, ciprofloxacin or chitosan+ciprofloxacin). The numbers of the samples in the pictures are referred to the group of samples explained in Scheme 1: group 1 was coated with chitosan, group 5 with ciprofloxacin and group 7 with a solution of both chitosan and ciprofloxacin. Group 11 and group 12 were first treated with plasma and coated with chitosan, but then chitosan coating in group 11 was neutralized, while group 12 coating was performed with a chitosan solution at a different $\mathrm{pH}$ (5.73).

Figure 5 shows the biological results for the bare and CS coated silicone tubes. In those cases the counting of bacterial colonies in all the dilutions assayed have displayed a bacterial growth close to that observed for the control samples was obtained in all the experimental 
groups studied. No statistically significant differences were observed among the control sample and the experimental groups. These results are in accordance with the XPS, FTIR and surface roughness data where the lack of an appropriated adherence between the CS and the silicone tubes was demonstrated. These results clearly show the paramount importance of the CVAD material in order to adhere and retain the coating on the surface of the device and so to achieve an efficient bactericidal action. The nature and roughness of the material used as substrate is crucial to obtain an adequate and functional coating against potential infections. The coating of CVADs has emerged as a potential tool in the biomedical field to minimize or even impair infections. These coatings are intended to solve the emergence and spread of nosocomial or chronic infections mainly initiated in the insertion site, based on different compounds such antibiotics or silver $58,10,11,18-21,32,59,60$. A recent work ${ }^{11}$ has described the surface modification of silicone rubber surfaces through a nanoscale plasma methodology using monomeric trimethylsilane and oxygen showing a significant inhibition of $S$. aureus biofilm formation in vitro which was dependent on the oxygen deposition ratio. The posterior in vivo study also revealed the efficiency of these devices in an infection model, mediated by the inoculation of $S$. aureus $\left(10^{8} \mathrm{CFU}\right)$, though a total reduction in the bacterial growth was not observed. The chemical immobilization of an antimicrobial peptide on the surface of gold nanoparticles has been successfully tested fully inhibiting $S$. aureus growth which could be translated onto other types of surfaces and medical devices ${ }^{59}$ though it is not so cost-effective and technologically affordable as CS. A nanostructured silver coating on polydimethylsiloxane surfaces deposited by using plasma enhanced chemical vapor deposition has been shown to fully diminish $S$. aureus viability without a significant decrease in COS-7 cell viability though linked to $\mathrm{Ag}^{+}$release ${ }^{10}$ which has been previously shown as potentially harmful ${ }^{23}$. 
CS has been widely shown as an effective in vitro bactericidal polymer against $S$. aureus ${ }^{38}$ displaying high biocompatibility on human cells. Our group has previously synthesized nanocomposites based on solid CS-gold-nanoparticle films exerting a total bactericidal effect in an in vitro infection model of $S$. aureus and P. aeruginosa at CS concentrations and Mws close to those described in this work, not showing cytotoxic effects against HaCaT and A549 cell lines ${ }^{61}$. Furthermore, the antibacterial effect of CS against other bacteria strains such as Escherichia coli has also been reported ${ }^{32,60}$. The deposition of CS alternatively with heparin onto aminolyzed poly(ethylene terephthalate) films to obtain bactericidal multilayer films showed a high efficiency after $24 \mathrm{~h}$ exerting a bacteria viability lower than $8 \%$ when $5 \times 10^{4}$ $\mathrm{CFU} / \mathrm{ml}$ were inoculated onto the multilayer surface. Interestingly, this effect was found to be dependent on the $\mathrm{pH}$ used during the assembly showing a higher efficiency at lower $\mathrm{pH}$ due to the changes in the degree of ionization of amino groups and CS, effect that was not revealed in our studies. In addition, these authors highlighted that this $\mathrm{pH}$ dependency is linked to the amount of CS deposited on the outer layer because most CS chains were observed at lower $\mathrm{pH}^{32}$. Moreover, the antibacterial activity of multifunctional composite coatings based on bioactive glasses and CS against E. coli was investigated in different surfaces $^{60}$. Though both bioactive glass and CS have demonstrated antibacterial properties, the composite coatings assayed exerted lower bactericidal effects than pure CS which was attributed to the differences in the surface roughness as it has been highlighted in our study. Some studies assessed the bactericidal mechanism by which CS is able to eradicate bacteria. The interaction with the negatively charged bacterial wall and/or membrane collapsing its functionality and inducing the cytoplasm components leakage ${ }^{62}$ is one of the proposed mechanisms of the biocidal action though other authors have pointed to CS nuclear mediated damage $^{38}$ or even the chelation of trace metals and the consequent disruption of the enzymatic activity ${ }^{63}$. 
The coating of CVADs with an efficient antimicrobial agent and non-toxic against human cells as CS makes this polymer a potential candidate for further applications in the prevention of chronic and nosocomial infections associated to medical devices.
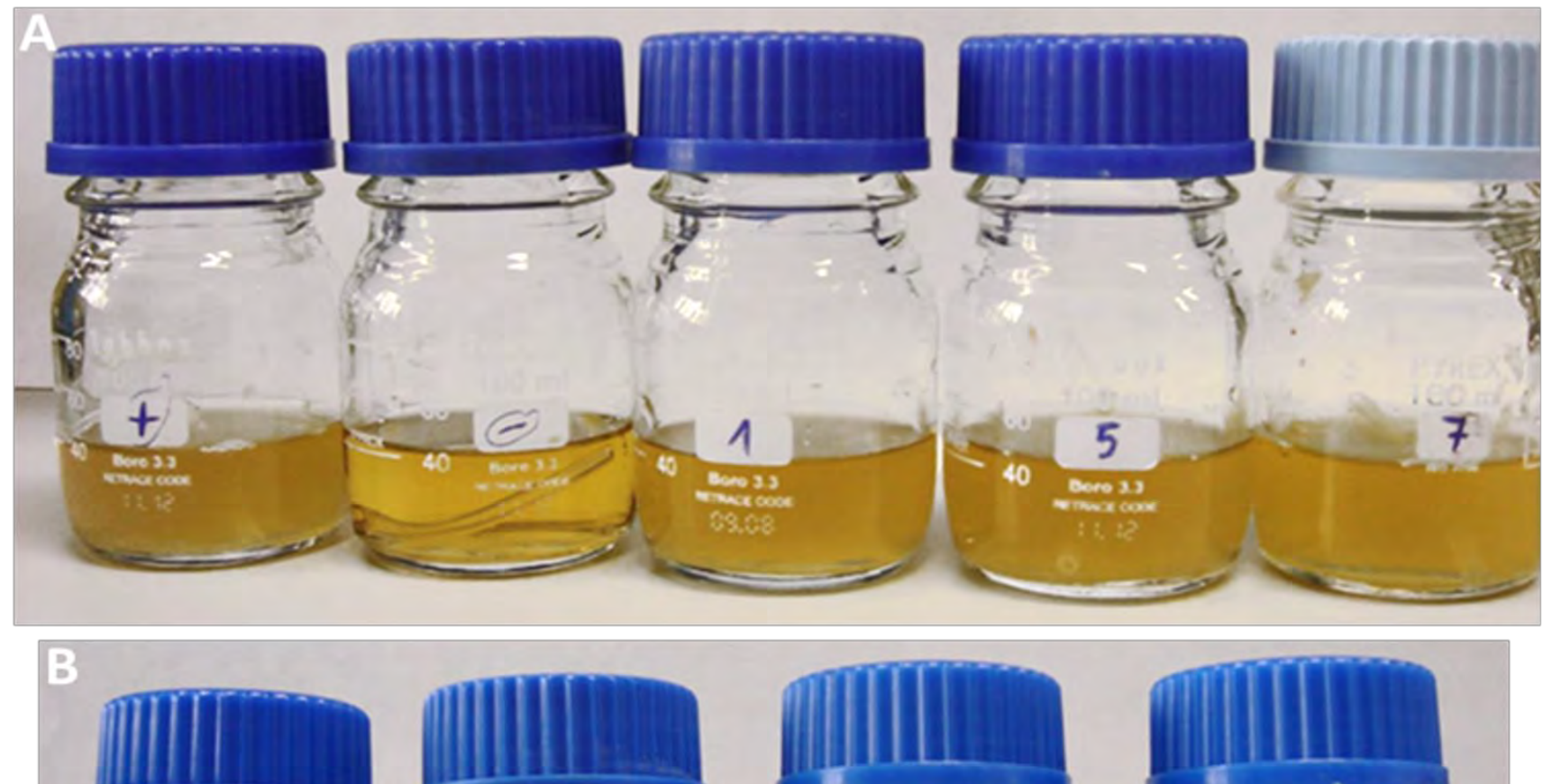
Figure 5. Silicon catheters (A, B) and colony forming units per $\mathrm{mL}(\mathrm{CFU} / \mathrm{mL})(\mathrm{C})$ obtained after treatment with $S$. aureus $\left(10^{2} \mathrm{CFU} / \mathrm{ml}\right)$. Positive and negative controls are shown (A, B). $S$. aureus grew in positive control due to the lack of coating and the treatment with the microorganism while the negative control was not treated with $S$. aureus showing that the sterilization process was successful for this type of materials. All the experimental groups displayed bacteria growth. The numbers of the samples in the pictures are referred to the group of samples explained in Scheme 1: group 1 was coated with chitosan, group 5 with 
ciprofloxacin and group 7 with a solution of both chitosan and ciprofloxacin. Group 11 and group 12 were first treated with plasma and coated with chitosan, but then chitosan coating in group 11 was neutralized, while group 12 coating was performed with a chitosan solution at a different $\mathrm{pH}(5.73)$.

In vitro biocompatibility tests

The in vitro cytotoxic effect of CS layers on HaCaT, A549 and CT26 cell lines was determined using extracts of materials. MTT and Alamar Blue assay performed using extracts with and without serum proteins revealed no cytotoxic effects (Figure 6). There was no statistical difference between the viability values after incubation with extracts with or without FBS. CS layers meet the requirement of the ISO 10993-5 norm (Biological evaluation of medical devices -- Part 5: Tests for in vitro cytotoxicity), which considers materials as non-cytotoxic when the cell viability is higher than $70 \%$.

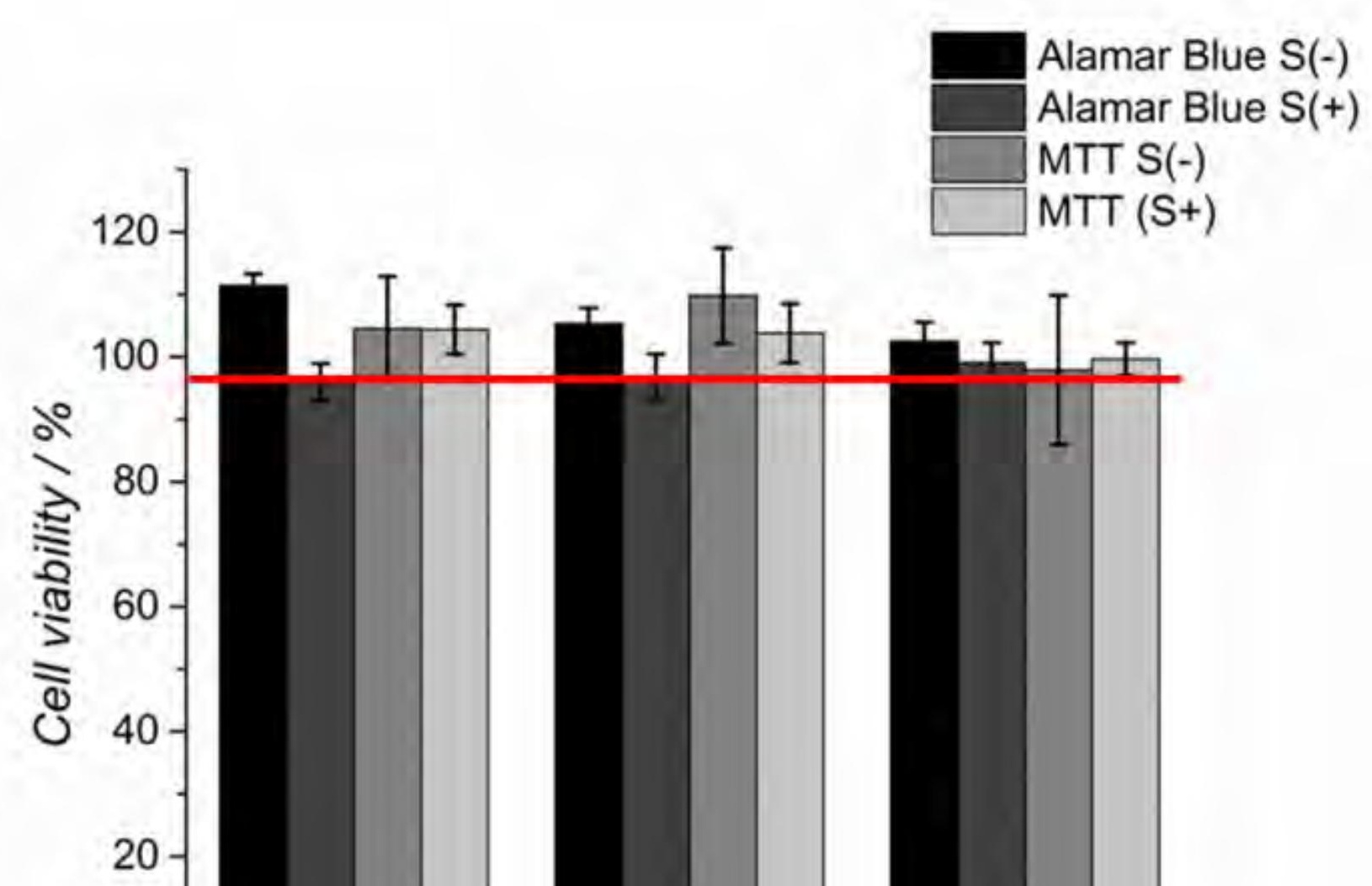


Figure 6. The cytotoxicity test results for the CS coatings towards three cell lines: human keratinocytes (HaCaT), human lung adenocarcinoma epithelial cell line (A549) and murine colon carcinoma cells (CT26). The red line marks the $100 \%$ cell viability which responds to the positive control (cells cultured in medium without sample). ( $\mathrm{S}(-)$ and $\mathrm{S}(+)$ stands for medium without and with serum proteins respectively upon the experiment performance).

\section{Conclusions}

CS coatings were successfully tested in 3D-printed reservoirs mimicking CVADs as powerful antimicrobial agents to avoid the development and spread of $S$. aureus infection showing high biocompatibility and low cytotoxicity. Our study indicated that the single presence of CS is enough to halt the infection being highly relevant the surface material regarding composition and roughness to obtain an efficient coating and thus the highest bactericidal activity. The addition of $\mathrm{AG}$ and/or plasma treatment did not improve the coating bactericidal efficiency as CS alone displayed a total bactericidal activity showing the same efficiency as the model antibiotic assayed. This work reveals the paramount importance of CS in the prevention of microbial colonization of CVADs underlining its potential as bactericidal agent in biomedical applications, though further studies regarding its hemostatic properties should be performed in order to fully understand its suitability for intravascular applications.

\section{Supplementary material}


Refer to Web version for supplementary material.

\section{Declaration of Conflicting Interests}

The authors declared no potential conflicts of interest with respect to the research, authorship, and/or publication of this article.

\section{Funding}

The authors are grateful for the financial support of the ERC Consolidator Grant program (ERC-2013-CoG-614715, NANOHEDONISM). CIBER-BBN is an initiative funded by the VI National R\&D\&i Plan 2008-2011, Iniciativa Ingenio 2010, Consolider Program, CIBER Actions and financed by the Instituto de Salud Carlos III (Spain) with assistance from the European Regional Development Fund.

\section{References}

1. Chauhan A, Ghigo J-M, Beloin C. Study of in vivo catheter biofilm infections using pediatric central venous catheter implanted in rat. Nat Protoc 2016; 11: 525-541.

2. Veenstra DL, Saint S, Saha S, et al. Efficacy of antiseptic-impregnated central venous catheters in preventing catheter-related bloodstream infection: a meta-analysis. JAMA $1999 ; 281: 261-7$.

3. Cheung E, Baerlocher MO, Asch M, et al. Venous access: a practical review for 2009. Can Fam physician Médecin Fam Can 2009; 55: 494-6.

4. Pittet D, Tarara D, Wenzel RP. Nosocomial bloodstream infection in critically ill patients. Excess length of stay, extra costs, and attributable mortality. JAMA 1994; 271: 1598-601.

5. Mermel LA. Prevention of intravascular catheter-related infections. Ann Intern Med 2000; 132: 391-402.

6. Baskin JL, Pui C-H, Reiss U, et al. Management of occlusion and thrombosis associated with long-term indwelling central venous catheters. Lancet (London, 
England) 2009; 374: 159-69.

7. Galloway S, Bodenham A. Long-term central venous access. Br J Anaesth 2004; 92: $722-34$.

8. Bayston R, Bhundia C, Ashraf W. Hydromer-coated catheters to prevent shunt infection? J Neurosurg 2005; 102: 207-12.

9. Bowler PG, Duerden BI, Armstrong DG. Wound Microbiology and Associated Approaches to Wound Management. Clin Microbiol Rev 2001; 14: 244-269.

10. Gilabert-Porres J, Martí S, Calatayud L, et al. Design of a Nanostructured Active Surface against Gram-Positive and Gram-Negative Bacteria through Plasma Activation and in Situ Silver Reduction. ACS Appl Mater Interfaces 2016; 8: 64-73.

11. $\mathrm{Xu} \mathrm{Y,} \mathrm{Jones} \mathrm{JE,} \mathrm{Yu} \mathrm{H,} \mathrm{et} \mathrm{al.} \mathrm{Nanoscale} \mathrm{Plasma} \mathrm{Coating} \mathrm{Inhibits} \mathrm{Formation} \mathrm{of}$ Staphylococcus aureus Biofilm. Antimicrob Agents Chemother 2015; 59: 7308-15.

12. Amorena B, Gracia E, Monzón M, et al. Antibiotic susceptibility assay for Staphylococcus aureus in biofilms developed in vitro. J Antimicrob Chemother 1999; 44: 43-55.

13. Monzón M, García-Alvarez F, Laclériga A, et al. Evaluation of four experimental osteomyelitis infection models by using precolonized implants and bacterial suspensions. Acta Orthop Scand 2002; 73: 11-9.

14. Gracia E, Laclériga A, Monzón M, et al. Application of a rat osteomyelitis model to compare in vivo and in vitro the antibiotic efficacy against bacteria with high capacity to form biofilms. J Surg Res 1998; 79: 146-53.

15. Monzón M, García-Alvarez F, Laclériga A, et al. A simple infection model using precolonized implants to reproduce rat chronic Staphylococcus aureus osteomyelitis and study antibiotic treatment. J Orthop Res 2001; 19: 820-6.

16. Kokai-Kun JF, Chanturiya T, Mond JJ. Lysostaphin eradicates established 
Staphylococcus aureus biofilms in jugular vein catheterized mice. J Antimicrob Chemother 2009; 64: 94-100.

17. Ryan JM, Ryan BM, Smith TP. Antibiotic prophylaxis in interventional radiology. $J$ Vasc Interv Radiol 2004; 15: 547-56.

18. Kamal GD, Pfaller MA, Rempe LE, et al. Reduced intravascular catheter infection by antibiotic bonding. A prospective, randomized, controlled trial. JAMA 1991; 265: $2364-8$.

19. Raad I, Darouiche R, Dupuis J, et al. Central venous catheters coated with minocycline and rifampin for the prevention of catheter-related colonization and bloodstream infections. A randomized, double-blind trial. The Texas Medical Center Catheter Study Group. Ann Intern Med 1997; 127: 267-74.

20. Taglietti A, Arciola CR, D'Agostino A, et al. Antibiofilm activity of a monolayer of silver nanoparticles anchored to an amino-silanized glass surface. Biomaterials 2014; 35: $1779-88$.

21. Sampath LA, Tambe SM, Modak SM. In vitro and in vivo efficacy of catheters impregnated with antiseptics or antibiotics: evaluation of the risk of bacterial resistance to the antimicrobials in the catheters. Infect Control Hosp Epidemiol 2001; 22: $640-6$.

22. Gallieni M, Brenna I, Brunini F, et al. Dialysis central venous catheter types and performance. J Vasc Access 2014; 15 Suppl 7: S140-6.

23. Nguyen KC, Richards L, Massarsky A, et al. Toxicological evaluation of representative silver nanoparticles in macrophages and epithelial cells. Toxicol In Vitro 2016; 33: 163-173.

24. Percival SL, Bowler PG, Russell D. Bacterial resistance to silver in wound care. $J$ Hosp Infect 2005; 60: 1-7. 
25. Sütterlin S, Edquist P, Sandegren L, et al. Silver resistance genes are overrepresented among Escherichia coli isolates with CTX-M production. Appl Environ Microbiol 2014; 80: 6863-9.

26. Chen Y-M, Dai A-P, Shi Y, et al. Effectiveness of silver-impregnated central venous catheters for preventing catheter-related blood stream infections: a meta-analysis. Int $J$ Infect Dis 2014; 29: 279-86.

27. Tan CP, Craighead HG. Surface Engineering and Patterning Using Parylene for Biological Applications. Materials (Basel) 2010; 3: 1803-1832.

28. Balazs DJ, Triandafillu K, Chevolot Y, et al. Surface modification of PVC endotracheal tubes by oxygen glow discharge to reduce bacterial adhesion. Surf Interface Anal 2003; 35: 301-309.

29. Choudhury AJ, Gogoi D, Kandimalla R, et al. Penicillin impregnation on oxygen plasma surface functionalized chitosan/Antheraea assama silk fibroin: Studies of antibacterial activity and antithrombogenic property. Mater Sci Eng C Mater Biol Appl 2016; 60: 475-84.

30. Pielesz A, Machnicka A SE. Antibacterial activity and scanning electron microscopy (SEM) examination of alginate-based films and wound dressings. Ecol CHEM ISTRY Eng S 2011; 18: 197-210.

31. Goh CH, Heng PWS, Huang EPE, et al. Interactions of antimicrobial compounds with cross-linking agents of alginate dressings. J Antimicrob Chemother 2008; 62: 105-108.

32. Fu J, Ji J, Yuan W, et al. Construction of anti-adhesive and antibacterial multilayer films via layer-by-layer assembly of heparin and chitosan. Biomaterials 2005; 26 : $6684-92$.

33. Dash M, Chiellini F, Ottenbrite RM, et al. Chitosan-A versatile semi-synthetic polymer in biomedical applications. Prog Polym Sci 2011; 36: 981-1014. 
34. Yi H, Wu L-Q, Bentley WE, et al. Biofabrication with chitosan. Biomacromolecules; 6: 2881-94.

35. Schiffman JD, Schauer CL. Cross-linking chitosan nanofibers. Biomacromolecules 2007; 8: 594-601.

36. $\mathrm{Mu} \mathrm{H}$, Guo F, Niu H, et al. Chitosan improves anti-biofilm efficacy of gentamicin through facilitating antibiotic penetration. Int J Mol Sci 2014; 15: 22296-308.

37. No H. Antibacterial activity of chitosans and chitosan oligomers with different molecular weights. Int J Food Microbiol 2002; 74: 65-72.

38. Rabea EI, Badawy ME-T, Stevens C V, et al. Chitosan as antimicrobial agent: applications and mode of action. Biomacromolecules; 4: 1457-65.

39. Ravi Kumar MN. A review of chitin and chitosan applications. React Funct Polym 2000; 46: 1-27.

40. Costa-Pinto AR, Martins AM, Castelhano-Carlos MJ, et al. In vitro degradation and in vivo biocompatibility of chitosan-poly(butylene succinate) fiber mesh scaffolds. $J$ Bioact Compat Polym 2014; 29: 137-151.

41. Machul A, Mikołajczyk D, Regiel-Futyra A, et al. Study on inhibitory activity of chitosan-based materials against biofilm producing Pseudomonas aeruginosa strains. $J$ Biomater Appl 2015; 30: 269-78.

42. Shalumon KT, Anulekha KH, Nair S V, et al. Sodium alginate/poly(vinyl alcohol)/nano $\mathrm{ZnO}$ composite nanofibers for antibacterial wound dressings. Int J Biol Macromol 2011; 49: 247-54.

43. Bumgardner JD, Wiser R, Gerard PD, et al. Chitosan: potential use as a bioactive coating for orthopaedic and craniofacial/dental implants. J Biomater Sci Polym Ed $2003 ; 14: 423-38$.

44. Raafat D, von Bargen K, Haas A, et al. Insights into the mode of action of chitosan as 
an antibacterial compound. Appl Environ Microbiol 2008; 74: 3764-73.

45. Wang X, Zhu K-X, Zhou H-M. Immobilization of glucose oxidase in alginate-chitosan microcapsules. Int J Mol Sci 2011; 12: 3042-54.

46. Wee S, Gombotz W. Protein release from alginate matrices. Adv Drug Deliv Rev 1998; 31: $267-285$.

47. Wang L, Shelton RM, Cooper PR, et al. Evaluation of sodium alginate for bone marrow cell tissue engineering. Biomaterials 2003; 24: 3475-81.

48. MOTWANI S, CHOPRA S, TALEGAONKAR S, et al. Chitosan-sodium alginate nanoparticles as submicroscopic reservoirs for ocular delivery: Formulation, optimisation and in vitro characterisation. Eur J Pharm Biopharm 2007; 68: 513-25.

49. Regiel A, Irusta S, Kyzioł A, et al. Preparation and characterization of chitosan-silver nanocomposite films and their antibacterial activity against Staphylococcus aureus. Nanotechnology 2013; 24: 15101.

50. Aegerter MA, Mennig M (eds). Sol-Gel Technologies for Glass Producers and Users. Boston, MA: Springer US. Epub ahead of print 2004. DOI: 10.1007/978-0-387-889535.

51. Mermel LA, Allon M, Bouza E, et al. Clinical practice guidelines for the diagnosis and management of intravascular catheter-related infection: 2009 Update by the Infectious Diseases Society of America. Clin Infect Dis 2009; 49: 1-45.

52. Butruk B, Ziętek P, Ciach T. Simple method of fabrication of hydrophobic coatings for polyurethanes. Open Chem; 9. Epub ahead of print 1 January 2011. DOI: $10.2478 / \mathrm{s} 11532-011-0094-7$.

53. Weyermann J, Lochmann D, Zimmer A. A practical note on the use of cytotoxicity assays. Int J Pharm 2005; 288: 369-76.

54. Arruebo M, Fernández-Pacheco R, Velasco B, et al. Antibody-Functionalized Hybrid 
Superparamagnetic Nanoparticles. Adv Funct Mater 2007; 17: 1473-1479.

55. Torabi S, Mahdavian AR, Sanei M, et al. Chitosan and functionalized acrylic nanoparticles as the precursor of new generation of bio-based antibacterial films. Mater Sci Eng C Mater Biol Appl 2016; 59: 1-9.

56. Khan IU, Serra CA, Anton N, et al. Continuous-flow encapsulation of ketoprofen in copolymer microbeads via co-axial microfluidic device: influence of operating and material parameters on drug carrier properties. Int J Pharm 2013; 441: 809-17.

57. Ranjha NM, Ayub G, Naseem S, et al. Preparation and characterization of hybrid pHsensitive hydrogels of chitosan-co-acrylic acid for controlled release of verapamil. $J$ Mater Sci Mater Med 2010; 21: 2805-16.

58. Gilbert RE, Harden M. Effectiveness of impregnated central venous catheters for catheter related blood stream infection: a systematic review. Curr Opin Infect Dis $2008 ; 21: 235-45$.

59. Rai A, Pinto S, Evangelista MB, et al. High-density antimicrobial peptide coating with broad activity and low cytotoxicity against human cells. Acta Biomater. Epub ahead of print 25 January 2016. DOI: 10.1016/j.actbio.2016.01.035.

60. Seuss S, Lehmann M, Boccaccini AR. Alternating current electrophoretic deposition of antibacterial bioactive glass-chitosan composite coatings. Int J Mol Sci 2014; 15 : $12231-42$.

61. Regiel-Futyra A, Kus-Liśkiewicz M, Sebastian V, et al. Development of noncytotoxic chitosan-gold nanocomposites as efficient antibacterial materials. ACS Appl Mater Interfaces 2015; 7: 1087-99.

62. Je J-Y, Kim S-K. Chitosan derivatives killed bacteria by disrupting the outer and inner membrane. J Agric Food Chem 2006; 54: 6629-33.

63. Cuero RG, Osuji G, Washington A. N-carboxymethylchitosan inhibition of aflatoxin 
production: Role of zinc. Biotechnol Lett 1991; 13: 441-444. 